\title{
MG13 proceedings: On the validity of the adiabatic approximation in compact binary inspirals
}

\author{
Andrea Maselli, Leonardo Gualtieri, Valeria Ferrari \\ Dipartimento di Fisica, "Sapienza", Università di Roma 8 Sezione INFN Roma1, Rome, Italy. \\ Francesco Pannarale \\ Max-Planck-Institut für Gravitationsphysik, Albert Einstein Institut, Potsdam, Germany.
}

\begin{abstract}
We use the post-Newtonian-Affine model to assess the validity of the adiabatic approximation in modeling tidal effects in the phase evolution of compact binary systems. We compute the dynamical evolution of the tidal tensor, which we estimate at the $2 \mathrm{PN}$ order, and of the quadrupole tensor, finding that their ratio, i.e. the tidal deformability, increases in the last phases of the inspiral. We derive the gravitational wave phase corrections due to this phenomenon and quantify how they affect gravitational wave detectability.
\end{abstract}

Keywords: Compact binary inspirals, tidal interactions, Love numbers

\section{Introduction}

Coalescing binary systems composed of neutron stars (NS) and/or black holes (BH) are one of the most promising sources of gravitational waves (GWs) for second and third generation of ground based interferometers [1]. Additionally, certain key features of NS tidal deformations may provide precious information about the NS equation of state (EOS) [2-5]. In current semi-analytical approaches, finite size effects are treated by assuming that the NS deformability is described in terms of a set of quantities, the Love numbers [6,7], among which the apsidal constant $k_{2}$ is the most important one for GW phenomenology. Within this framework and under the so called adiabatic approximation, the evolution of a star embedded in an external quadrupolar tidal field, $C_{i j}$, is governed by the equation

$$
Q_{i j}=-(2 / 3) k_{2} R_{\mathrm{NS}}^{5} C_{i j}
$$

where $Q_{i j}$ and $R_{\mathrm{NS}}$ are the star quadrupole moment and radius at isolation, respectively.

In this work, on the basis of the results of Maselli et al. 2012 [8], we employ the post-Newtonian-Affine model (PNA) [9], recently developed to study tidal interactions in BH-NS and NS-NS systems, to asses the range of validity of Eq. (11). By computing the ratio $\sim Q_{i j} / C_{i j}$ as function of the orbital separation for several binary configurations, we find that $k_{2}$ is not constant during the inspiral and that it grows in the last stages of the inspiral. Finally, we estimate the impact of such effect on future GWs detections.

\section{The PNA model}

To describe stellar deformations, we improve the PNA model, based on the Affine approach [10-14], which relies on the assumption that a NS in a binary system 
is deformed into an ellipsoid by the tidal forces exerted by its companion. Under the Affine hypothesis, the infinite degrees of freedom of the stellar fluid reduce to five dynamical variables, namely the three principal axes of the ellipsoid and two angles related to the star angular velocity and the internal fluid motion. The main improvements to the original Affine approach [10-12], were brought in $[9,13,14]$, where the NS spherical equilibrium configuration was determined by solving the relativistic stellar structure equations, and a more careful treatment of coordinate systems and of relativistic corrections to the star internal dynamics were introduced. Furthermore, starting from a two-body post-Newtonian metric $[15,16]$, an explicit expression for the tidal field was derived. This represents a major change from the original approach in which the NS moves in a background space-time described by the Kerr metric, and it allows to consistently treat stellar deformations in NS-NS binaries. The orbital motion is handled within a post-Newtonian framework, as well: the phase evolution is determined by means of the TaylorT4 approximant [17], including the effects of tidal interactions on the orbital motion, up to $1 \mathrm{PN}$ order [18].

The source of stellar deformations in the PNA model is given by the quadrupolar tidal tensor $C_{i j}=R_{\alpha \beta \gamma \delta} e_{(0)}^{\alpha} e_{(i)}^{\beta} e_{(0)}^{\gamma} e_{(j)}^{\delta}$, where $R_{\alpha \beta \gamma \delta}$ is the Riemann tensor of the $3 \mathrm{PN}$ metric describing the orbital motion, and $e_{(i=0, \ldots, 3)}$ is an orthonormal tetrad field fixed to the star center of mass and parallel transported along its motion. The results obtained in [9], where we derived $C_{i j}$ up to $\mathcal{O}\left(1 / c^{3}\right)$, were extended in [8] to include $\mathcal{O}\left(1 / c^{4}\right)$ terms. With this aim, we also computed the $2 \mathrm{PN}$ contribution to the spatial tetrad vectors $e_{(k)}^{j}$ introduced in [19]. $C_{i j}$ depends on the masses of both compact objects $m_{1,2}$, and includes, $1.5 \mathrm{PN}$ order, the spin contribution from rotating objects.

\section{Tidal Love number evolution}

We solve the PNA equations of motion up to the onset of the NS mass shedding, for a representative set of binary system configurations, by considering two equations of state which are expected to cover the range of possible NS deformabilities. For each binary model, we compute $k_{2}$ as the ratio between the quadrupole and the tidal tensor, from Eq. (11). We find that this quantity is a function of the orbital separation $r$, i.e. $k_{2}=k_{2}(r)$, that increases during the binary inspiral by a $\sim 10 \%-30 \%$ factor. The constant Love number $\bar{k}_{2}$ should be regarded as the asymptotic limit of this function, i.e. $\bar{k}_{2}=\lim _{r \rightarrow \infty} k_{2}(r)$. This effect is strongly dependent on the EOS choice: it is larger/smaller for a less/more compact NS. The dependence of this effect on the mass ratio is weaker, and it increases more for larger values of $q=m_{2} / m_{1} \leq 1$. This effect appears to be nearly insensitive to the NS mass.

Replacing the Love function $k_{2}(r)$ into the expression of the GW signal, we estimate a new correction to the GW phase. This adds linearly to the point-particle (PP) term, and to the tidal term (T) computed by assuming the Love number to be constant:

$$
\psi_{\mathrm{GW}}=\psi_{\mathrm{PP}}+\psi_{\mathrm{T}}+\delta \psi_{T},
$$


where $\delta \psi_{T}$ describes the growth of $k_{2}(r)$ during the inspiral. We find that the new contribution is always less than 1 radian for all considered configurations, up to the onset of mass shedding.

To assess the validity of the adiabatic approximation as far GW detectability is concerned, we compute overlaps [20] between PP templates and real signals which take into account tidal effects at the best of our knowledge, i.e. including $\delta \psi_{T}$. We find that in the worst case, i.e. for a NS-NS binary with a standard total mass of $2.8 M_{\odot}$, the number of missed events due to the use of PP templates could be as high as $11 \%$ and $13 \%$ for the second and third generation of ground based detectors, respectively, depending on the NS equation of state. By using templates which include tidal corrections by means of the constant Love number, however, the overlaps would differ from unity by less than a part in one thousand for all configurations considered. We may thus conclude that building gravitational waveforms within the adiabatic approximation is very reliable up to the mass-shedding.

\section{Conclusions}

In this work we reviewed the results of [8], in which we improved the PNA model by computing the $2 \mathrm{PN}$ contribution to the post-Newtonian tidal tensor. We used this framework to assess the reliability of the Love number adiabatic approximation and found that the NS deformability increases during the last phases of the inspiral up to $30 \%$, depending on the NS EOS.

\section{References}

1. http://www.ego-gw.it; http://www.ligo.caltech.edu; http://www.et-gw.eu/.

2. E.E. Flanagan, T. Hinderer, Phys. Rev. D 77, 021502 (2008).

3. T. Hinderer, B.D. Lackey, R.N. Lang, J.S. Read, Phys. Rev. D 81, 123016 (2010).

4. T. Damour, A. Nagar, Phys. Rev. D 80, 084035 (2009).

5. F. Pannarale, L. Rezzolla, F. Ohme and J. S. Read, Phys. Rev. D 84, 104017 (2011).

6. T. Hinderer, Astrophys. J. 677, 1216 (2008); ibid., 697, 964 (2009).

7. T. Binnington, E. Poisson, Phys. Rev. D 80, 084018 (2009).

8. A. Maselli, L. Gualtieri, F. Pannarale and V. Ferrari, Phys. Rev. D 86, 044032 (2012).

9. V. Ferrari, L. Gualtieri, A. Maselli, Phys. Rev. D 85, 044045 (2012).

10. B. Carter, J.P. Luminet, Mon. Not. R. Astron. Soc. 212, 23 (1985).

11. J.P. Luminet, J.A. Marck, Mon. Not. R. Astron. Soc. 212, 57 (1985).

12. P. Wiggins, D. Lai, Astrophys. J. 532, 530 (2000).

13. V. Ferrari, L. Gualtieri, F. Pannarale, Class. Quant. Grav. 26, 125004 (2009).

14. V. Ferrari, L. Gualtieri, F. Pannarale, Phys. Rev. D 81, 064026 (2010).

15. L. Blanchet, G. Faye, and B. Ponsot, Phys. Rev. D 58, 124002 (1998).

16. G. Faye, L. Blanchet, and A. Buonanno, Phys. Rev. D 74, 104033 (2006).

17. L. Santamaria et al., Phys. Rev. D 82, 064016 (2010).

18. J. Vines, E.E. Flanagan, T. Hinderer, Phys. Rev. D 83, 084051 (2011).

19. T. Fukushima, Cel. Mech. and Dynamical Astron. 44, 61 (1988).

20. L. Lindblom, B.J. Owen, and D.A. Brown, Phys. Rev. D 78, 124020 (2008). 